\title{
La route en train de se faire...
}

\section{Nicolas Verdier et Sandrine Robert}

Comment la route était-elle pensée par les acteurs des temps passés ?. Le passage par le vocabulaire nous en donne quelques indices. En effet, on le sait, le mot route vient du latin rupta, participe passé de rumpere. Le mot désigne dans son sens premier une rupture et appartient au vocabulaire du défrichement. Il apparaît en France au XIe siècle au moment de l'extension des zones cultivées et peut signifier aussi bien un petit sentier de forêt qu'une bande de gens de guerre (Froissart). Le mot signifie aussi bien rompre, dans le sens de rompre un ordre dans la forêt pour imposer une humanisation par la voie de communication. Le mot qui, au Moyen-Âge, se rapproche le plus de celui de route, tel qu'il a été décrit ici, est celui de camin, ou chemin. Il est très proche de cheminer et place le mot chemin dans un entre-deux, entre pratiques du déplacement et sédimentation sur le sol. Ce qui est mis ici en avant ne relève donc ni de l'approche morphologique, ni de l'approche fonctionnelle. Nous sommes plus ici du côté d'une description génétique qui donne à voir le vocabulaire de la voie de communication en train de se constituer. Le retour des pratiques remet en cause tant la seule sédimentation que la seule fonction. C'est là que des objets qui intégraient difficilement les deux premières conceptions trouvent le plus aisément leur place. En effet, on connaît depuis longtemps les cas de "dédoublement" de voies, voire les "systèmes de doublets d'une même route" (Loubès. 1982, Grenier 1934); d'ailleurs, qui n'a jamais utilisé un itinéraire de délestage lors de ses pérégrinations estivales ? Chacun des auteurs de ce dossier, du plus théorique au plus empirique a été amené à évoquer cette question. Sandrine Robert dans son texte sur la route-monument, parle ainsi de "route faisceau", Nicolas Verdier traite des “vibrations du réseau", Claire Marchand s'intéresse aux "mobilités des tracés",. Sur un autre registre, le second article de Sandrine Robert montre les suites ininterrompues d'usages, d'abandons, de reprises, de transformations et de transmissions. Stéphane Blond montre bien au travers des planches de l'Atlas Trudaine la coexistence d'anciens et de nouveaux tracés. Jean-François Gleyze montre bien quant à lui l'intérêt de ne pas tenir compte d'un chemin unique, même s'il est le plus court, entre deux lieux. Enfin l'article d'Arnaud Chevalier, montre la coexistence, de deux chemins parallèles pour un même itinéraire. Le cœur du dossier se trouve ici, dans cette co-présence de voies qui oblige à penser des usages et des 
pratiques diverses et des chronologies complexes.

Sur le terrain ou dans les sources coexistent donc plusieurs tracés, souvent de niveaux différents, entre deux lieux. Dans l'approche typo-chronologique, ces doublets intègrent des niveaux hiérarchiques différents. Une limite de cette approche apparaît ici puisqu'elle mène à penser une hiérarchie routière uniquement liée aux dimensions, à la forme ou aux matériaux utilisés. Elle refuse d'imaginer la simple coexistence à un même niveau, quelle que soit la forme. Dans l'approche fonctionnaliste, ces différentes voies sont la même, et n'ont pas d'intérêt en tant que tel. Ce qui importe c'est que la communication existe. L'échelle de prise en compte de la route est celle du flux entre les lieux où se concentrent les fonctions.

Passer par une approche génétique permet de dépasser les limites des raisonnements typo-chronologiques, et fonctionnels. Cela invite à dépasser l'idée d'une sédimentation unique pour en arriver à des phases qui se succèdent en fonctions de temporalités complexes. Dès lors, la durée prend un sens nouveau et le réseau transmis n'est plus romain, ou gaulois, voire médiévale ou moderne. Chaque période innove soit, mais en s'appuyant sur l'existant, en se l'appropriant parfois pour des usages imprévus. De nombreux cas ont été démontrés pour l'architecture moderne et contemporaine, à travers la réappropriation de quartiers entiers qui transmettent les formes tout en les transformant (Castex et al. 1979 par exemple) ou d'architectures particulières comme l'Hôtel aristocratique parisien au XVIIIe siècle qui passe de l'aristocratie aux ministères, voire à l'habitation en appartements (Coquery. 1998). Dans cela rien n'était prévu mais la morphologie a été appropriée, parfois à l'échelle la plus fine. -

Dans le cas des réseaux viaires, la question des échelles est d'ailleurs des plus nécessaires (Verdier. 2004). Les interventions et adaptations pratiquées à une échelle ne sont pas les mêmes à une autre échelle. Dès lors c'est l'ensemble de l'interrelation de ces échelles qui nécessite d'être reprise, à nouveau frais, à chaque moment du réseau. On notera de ce point de vue que la plupart des auteurs de ce dossier ont ressenti la nécessité de distinguer au moins deux échelles, et parfois plus, opposant au minimum le local, ou le terrain, au régional, voire au supra-régional. À certaines échelles, les approches typo-chronologiques et morphologiques semblent dominer, à d'autres, c'est l'approche fonctionnelle, le tout dans une série de rejeux constants. Une route locale n'est pas toujours qu'une route locale, ce que par exemple l'approche hiérarchique nous empêche de saisir. En d'autres mots, aller chercher son pain en empruntant une route nationale ne fait pas de ce trajet, un trajet national. Inversement le réseau national peut se constituer par le bas, un peu à la façon dont les frontières nationales se construisent peut-être d'abord dans les villages frontaliers (Sahlins. 1996). Dès lors la question du rôle du pouvoir central, toujours évoquée lorsque l'on traite des voies de 
communication, se trouve en partie disqualifiée. En partie seulement car il semble que lorsqu'un pouvoir fort en a la possibilité, il tente d'organiser le réseau, voire l'étend ; mais même sans pouvoir fort, les transmissions et les réappropriations ont lieu $\div$ l'ensemble des voies se maintient. Ce qui disparaît peut-être alors, c'est le réseau, c'est-à-dire le système qui permet de penser l'ensemble comme un tout (Verdier, 2007).

La route ne serait donc pas à prendre comme un tracé unique et stable menant d'un point à un autre, mais plutôt comme un espace route composé de plusieurs faisceaux utilisés à une même époque ou pas, une aire de routes plus ou moins parallèles et de tailles variées, dont l'usage peut se trouver déterminé par les saisons, l'état de la voirie et les nécessités des voyageurs, voire par les moments où l'usage s'intensifie ou se restreint. Vidal de la Blache (1902) et Lucien Febvre (1922) s'entendent sur une même définition de cette "chose presque immatérielle qu'on appelle une voie de communication". On retrouve là la question des voies multiples. La fin de cet espace-voie de communication, serait alors liée à l'affirmation de l'État par la route. On aurait alors, d'abord une labilité, puis une fixation ? Soit, mais dans le même temps, comme pour redonner raison à l'approche morphologique, ces voies ne changent parfois que très peu de localisation. La route apparaît résiliente. À la façon de Galilée, on pourrait dire : et pourtant elles durent... La voie de communication est fragile, labile, variable, et cependant elle se retrouve bien souvent à la même place. Il y a là tout de l'oxymore : l'instabilité et la variabilité, mais au même endroit. En archéogéographie, les concepts de transformission, isotopie, isoclinie et iso-axialité rendent compte de ces phénomènes observés également pour les trames parcellaires (Chouquer 2000). Là se réunissent toutes les difficultés du déterminisme et du fonctionnalisme. Ce n'est probablement que par une approche génétique qui accepte de faire varier les échelles accordant aux sédimentations, aux fonctions, aux variations chacune les leurs que l'on peut décomposer un tel objet pour le rendre intelligible. 


\section{Bibliographie du dossier « Du sentier à la route. Une archéologie des réseaux viaires », coordonné par Sandrine Robert et Nicolas Verdier dans les Nouvelles de l'Archéologie n 115}

Agache, R. 1978. La Somme pré-romaine et romaine. Amiens, Société des Antiquaires de Picardie, $515 \mathrm{p}$.

Allen, W. B., Liu, D., Singer, S. 1993. «Accessibility measures of US metropolitan areas », Transportation Research, 27B (6) : 439-449.

Les Annales, 1989., “Tentons l’expérience ”, Annales E.S.C. nº. 1317-1323.

Antorne A. 2000. Le paysage de l'historien, Archéologie des bocages de l'Ouest de la France à l'époque moderne, Rennes, Presses universitaires de Rennes. 344 p.

Antoine, A., Vennel J.-P. 1994. «La voie royale et le paysage », Société archéologique et d'histoire de la Mayenne, fascicule 17. 35-57.

Arbellot G. 1973. « La grande mutation des routes de France au milieu du XVIII ${ }^{\mathrm{e}}$ siècle », Annales Économies Sociétés Civilisations, n³ : 765-791.

Arbellot, G. 1980. « Le réseau des routes de poste, objet des premières cartes thématiques de la France moderne », 104e congrès des sociétés savantes, Bordeaux 1979, Histoire moderne, Paris, Bibliothèque Nationale, $1: 97-115$.

Auray, J.-P., Bailly, A., Derycke, P.-H., Huriot, J.-M. 1994. Encyclopédie d'économie spatiale Concepts - Comportements - Organisations. Economica, Paris, 427 p.

Bairoch, P., Batou, J. et ChÈvre, P. 1988. La population des villes européennes de 800 à 1850, Genève, Droz.

Balard, M. dir., Laurioux, B. Le Jan, R., Le Mené, M. 2003. Dictionnaire de la France médiévale. Paris, Hachette, 287 p.

Bautier, R. H. 1987. « La route française et son évolution au cours du Moyen Age », Académie royale de Belgique, Bulletin de la classe des lettres et sciences morales et politiques, 1987, t. 73 : 70-104.

Benort, F. 1998. Modes d'occupation de l'espace protohistorique dans le Bassin moyen de l'Aisne, Mémoire de Maîtrise d'Archéologie, Université Panthéon-Sorbonne Paris I, 252 p. 
Berge, C. 1973. Graphes. Éditions Gauthier-Villars, Paris, 400 p.

Berger, J. F., Bertoncello, F. , Braemer, F., Datvian, G., Gazenbeck, M. (dir.) 2005. Temps et Espaces de l'Homme en société, analyses et modèles spatiaux en archéologie. XXVe rencontres internationales d'archéologie et d'histoire d'Antibes, Antibes, Editions APDCA, 543 p.

Bertrand, A.1864. «Voies romaines en Gaule, voies des itinéraires. Résumé du travail de la Commission de la topographie des Gaules », Extrait de la Revue archéologique. Paris, Librairie académique, $61 \mathrm{p}$.

Bradley, R. 1991. "The pattern of change in British prehistory », in : T. Earle (ed.), Chiefdoms: power, economy and ideology. Cambridge : 44-70.

Bradley, R. et Hart, C. 1983. "Prehistoric Settlement in the Peak District during the Third and Second Millennia bc: A Preliminary Analysis in the Light of Recent Fieldwork », Proceedings of the Prehistoric Society, 49 : 177-193.

BRÉCHON, F. 2000. Réseau routier et organisation de l'espace en Vivarais et sur ses marges au MoyenAge. Thèse d'histoire. Lyon, Université de Lumière Lyon 2, 1368 p.

Bretagnolle, A. et Verdier, N. 2007. «L'extension du réseau des routes de poste en France de 1708 à 1833 », in Le Roux, M. (dir.), Postes d'Europe XVIIIe-XXIe siècles. Jalons d'une histoire comparée, Paris, Comité pour l'Histoire de la Poste, 2007, 155-171.

BRUAND, O. 2002. Voyageurs et marchandises aux temps carolingiens: les réseaux de communication entre Loire et Meuse aux VIIIe et IXe siècles. Bruxelles, De Boeck Université, 357 p.

Bruinsma, F., Rietveld, P. 1993. «Urban agglomerations in European infrastructure networks », Urban Studies, 30 : 919-934

Brun, P., Guichard, Y. et Le Goff, I. 2005. « Les tombes à incinération de l'âge du Bronze et du Ier âge du Fer dans le bassin de l'Aisne : Observations préliminaires. ", in : Les pratiques funéraires à l'âge du Bronze en France, Actes de la table ronde de Sens-en-Bourgogne, 10-12 juin 1998. Société Archéologique de Sens, Comité des travaux historiques et scientifiques : 477-492.

Burgess, C. 1980. The Age of Stonehenge. Londres, J.M. Dent \& Sons Ltd, 402p.

CАРOT-Rey, R. 1946. Géographie de la circulation sur les continents, Paris, Gallimard, 296 p.

Castex, J, Céleste P. et Panerai Ph. 1979. Lecture d'une ville : Versailles. Paris, Editions du Moniteur, 
col. Architecture "Etudes", 235 p.

Cattan, N., Grasland, C. 1997. Les différentiels d'accessibilité des villes moyennes en France. Rapport de recherche pour le METL, CNRS, Laboratoire ParisGeo, 115p.

Chabaud, G., Cohen, E., Coquery, N. et Penez J. 2000. Les guides imprimés du $16^{\text {ème } a u ~} 20^{e ̀ m e}$ siècle, villes, paysages voyages, Paris, Belin, $704 \mathrm{p}$.

Chapelon, L. 1997. Offre de transport et aménagement du territoire - Evaluation spatio-temporelle des projets de modification de l'offre par modélisation multi-échelles des systèmes de transport. Thèse de doctorat en Aménagement, Université François Rabelais de Tours, 558 p.

Chapelot, J. 1993. "L'habitat rural : organisation et nature », in L'Ile-de-France, de Clovis à Hugues Capet, du Ve au Xe siècle, catalogue de l'exposition, Guiry-en-Vexin, Musée archéologique, 178-199.

Снаротат, G. 1981. « La voie protohistorique sud de la croisée de Vienne. Essai de reconstitution de son tracé jusqu'à Marseille ». Revue Archéologique de l’Est, 1981, Tome XXXII, n¹25-126 : 83-91

Chartrain, A. 2007. «Parcellaires fossiles et faits funéraires dans l'espace et le temps sur le territoire rural de Montpellier » Medieval Europe Paris 2007, http://www.archeogeographie.org

Chevalier, A. 2005. Les enclos funéraires protohistoriques de Basse-Normandie : Essai d'analyse spatiale, Mémoire de Maîtrise d'Archéologie, Université Panthéon-Sorbonne, Paris I. 175 p.

CHevalier, A. 2006. Recherche d'une structuration de l'espace protohistorique en Basse-Normandie : exercice d'analyse spatiale, Mémoire de Master II Recherche, Université Panthéon-Sorbonne, Paris I. $125 \mathrm{p}$.

Chevallier, R. 1972. Les voies romaines. Paris, Armand Colin, 313 p.

Chevallier, R. 2000. Lecture du temps dans l'espace. Topologie archéologique et historique Paris, Picard, 229 p.

Chevallier, R. dir. 1983. Les voies anciennes en Gaule et dans le monde romain occidental, Colloque tenu à Paris, ENS, 5-6 juin 1982 ». Caesarodunum nXVIII, Tours, Université de Tours, 487 p.

Chouquer, G. (dir.) 1996. Les Formes du paysage, Tome I : études sur les parcellaires : pré-actes du colloque d'Orléans (mars 1996). Paris, éditions Errance, 222 p.

Chouguer, G. (dir.) 1996. Les Formes du paysage, Tome.2: Archéologie des parcellaires, Paris, 
Editions Errance, 263 p.

Chouquer, G. (dir.) 1997. Les Formes du paysage, Tome 3 : L'analyse des systèmes spatiaux, Paris, Editions Errance, 198 p.

Chouquer, G. 1996. «Parcellaires et longue durée; Points de repères historiques et problèmes d'interprétation", in: Chouquer G. dir. Les Formes du paysage, Tome 2 : Archéologie des parcellaires. Actes du colloque d'Orléans (mars 1996). Paris, Editions Errance, 1996 : 201-223.

Chouquer, G. 2000. L'étude des paysages. Essais sur leurs formes et leur histoire. Editions Errance. Paris. 208 p.

Chouquer, G. 2006. «L'émergence de la planimétrie agraire à l'Age du Fer », in : Chouquer G. dir. Etudes Rurales $\mathrm{n}^{\circ} 175-176: 29-52$.

Chouquer, G. et al. 1996. « La morphologie agraire et les paysages de la plaine des Tilles et de l'Ouche (Côte-d'Or)». Chouquer, G. (dir.) Les Formes du paysage, Tome I : études sur les parcellaires : préactes du colloque d'Orléans (mars 1996). Paris, éditions Errance,: 32-49.

Coquery, N. L'Hôtel Aristocratique. Le marché du luxe à Paris au XVIIIe siècle, Paris, Publications de la Sorbonne. $444 \mathrm{p}$.

Dainville F. (de) 1964. Le langage des géographes, termes signes, couleurs des cartes anciennes, 1500-1800, Paris, Picard. XX-384 p.

Daremberg, Ch. et Saglio, E. 1969. Dictionnaire des antiquités grecques et romaines : d'après les textes et les monuments. Paris, Hachette, pp. écrit entre 1877 et 1906, 10 vol. $1^{\text {ère }}$ édition 1934.

Daveau, I. et Yvinec, J. H. 2002. «L'occupation gallo-romaine du site de Fontenay-en-Parisis "La Lampe" (Val-d'Oise) : établissement agricole spécialisé ou lieu de culte ?» in : Revue Archéologique du Centre, Tome 41, Tours, $2002: 129-172$.

Decoupigny, C. 2003. «Couloirs de trafics et émissions de polluants », in: Mathis, Ph. (dir.) 2003. Graphes et réseaux, modélisation multiniveau. Paris, Lavoisier: 187-211.

Demangeon, P. 1995. La Poste en Basse Normandie, Vire, Charles Corlet éditions. 439 p.

DenFord, G.T. 1975. « Economy and location of Bronze Age "arable" settlements on Dartmoor », Bulletin of the Institute of Archaeology, 12 : 175-196. 
Derville, A. 1978. «La première révolution des transports continentaux, (c.1000 - c. 1300.)», in : Annales de Bretagne et des Pays de l'Ouest 85 (1978) : 181-205

Desbordes, J. M. et Barrière, B. 1983. «Anciens itinéraires entre Limousin et Périgord », in : Chevallier, R. dir. 1983. «Les voies anciennes en Gaule et dans le monde romain occidental, Colloque tenu à Paris, ENS, 5-6 juin 1982 ». Caesarodunum $n^{\circ} X V I I I$, Tours, Université de Tours, : 189-199.

Desloges, J. à paraître. Histoire du peuplement de la Basse-Normandie, L'apport de la prospection aérienne 1988 - 2004. Direction Régionale des Affaires Culturelles de Basse-Normandie, Service Régional de l'Archéologie.

Devroey, J. P. 1984. «Un monastère dans l'économie d'échanges : les services de transport à l'abbaye Saint-Germain-des-Prés au IXe siècle », in : Annales Economie Sociétés Civilisation, 39, 1984 : 570589

Dion, R. 1961. Histoire des levées de la Loire, Paris, 312 p.

Duindian, F. 1991. Méthodes pour l'archéologie. Paris, Armand Colin, 401 p.

Dunin-Wasowicz, T. 1982. «Les routes médiévales en Europe centrale : bilan et perspectives », in :

Durand-Dastès, F. et al. 1998. Archaeomedes. Des oppida aux métropoles. Archéologues et géographes en vallée du Rhône. Paris, Anthropos, Economica, 280 p.

EJstrud, B. 2005. «Cost surface analysis and ancient roads : a comparison », in : Berger, J. F., Bertoncello, F. , Braemer, F., Datvian, G., Gazenbeck, M. (dir.) 2005. Temps et Espaces de l'Homme en société, analyses et modèles spatiaux en archéologie. XXVe rencontres internationales d'archéologie et d'histoire d'Antibes, Antibes, Editions APDCA : 135-139.

FAVIER, J. 1993. Dictionnaire de la France médiévale. Paris, Fayard, 982 p.

Favory, F. , Fiches, J.L., Raynaud, C. et Clatot, M. « La dynamique de l'habitat gallo-romain dans la basse vallée du Rhône », in DuRAnd-Dastès, F.et al. 1998. Archaeomedes. Des oppida aux métropoles. Archéologues et géographes en vallée du Rhône. Paris, Anthropos, Economica : 73-115.

Favory, F., Parodi, A., Poupet, P. et Raynaud, C. 1994. « Lunel-Viel et son territoire », in : Favory F. et Fiches J. L. (dir.). Les campagnes de la France méditerranéenne dans l'Antiquité et le haut MoyenAge, DAF n², Paris, Editions de la maison des sciences de l’Homme, 1994 : 163- 244 
Febvre, L. 1922. La terre et l'évolution humaine, introduction géographique à l'histoire, Paris, Renaissance du livre, $470 \mathrm{p}$.

Fourquin, G. 1964. Les campagnes de la région parisienne à la fin du Moyen-Âge, Paris, PUF, 590 p.

Freeman, L. 1979. «Centrality in social networks - Conceptual clarification ». Social Networks, $\mathrm{n}^{\circ} 1$ : 215-239

Garmy, P., Kaddouri, L., Rozenblat C. et Schneider, L. 2005 « Logiques spatiales et « systèmes de villes » en Lodévois de l'Antiquité à la période moderne », in BERGER, et al. (dir.). Temps et Espaces de l'Homme en société, analyses et modèles spatiaux en archéologie. XXVe rencontres internationales d'archéologie et d'histoire d'Antibes, Antibes, Editions APDCA : 225-236.

Gleyze, J.-F. 2005. La vulnérabilité structurelle des réseaux de transport. Thèse de doctorat de Géographie - Université de Paris VII Denis Diderot, 848 p.

Gleyze, J.-F. 2007a. «Effets spatiaux et effets réseaux dans l'évaluation d'indicateurs sur les nœuds d'un réseau d'infrastructures ». CyberGeo, n॰370, 24 p. http://www.cybergeo.eu/

GLEYZE, J.-F. 2007b. « Reducing functional network vulnerability - Structural indicators to anticipate the structural reorganization of a damaged transportation network », in : Actes de la 15ème édition de la Conférence Européenne de Géographie Théorique et Quantitative - ECTQG, Montreux, Suisse, 4 p.

GoHIER, Th. 1997. La poste aux chevaux dans la région malouine (1738-1870), Thèse de doctorat de Sciences Sociales de l'universtié de Haute-Bretagne Rennes II, sous la direction de Claude Nières, 1997, 2 vol.

Gould, S.J. 2005. Le renard et le hérisson, Comment combler le fossé entre la science et les humanités. Trad. N. Witkowski, Paris, Seuil, 364 p.

GrenIER, A. 1985. Manuel d'archéologie gallo-romaine. Tome 2, L'archéologie du sol. Navigationoccupation du sol. Paris, Editions Picard, 468 p. 1 ère édition 1934.

Groupe Dupont, 2004, Géopoint 2004, La forme en géographie, Avignon, Groupe Dupont, 505 p.

Guilaine, J. 1991. «Vers une préhistoire agraire » in: J. GuILAINE (dir.), Pour une archéologie agraire, Paris, Armand Colin : 31-80. 
Guilaine, J. 2000. « Changeons d'échelles : pour la très longue durée, pour de larges espaces », Etudes rurales, 153-154:9-21.

Guillaumet, J. P. 1996. L'artisanat chez les gaulois. Paris, Errance, 127 p.

Guillerme, A., 1984, Corps à corps sur la route. Les routes, les chemins et l'organisation des services du XIXe siècle, Paris, Presses de l'ENPC, 172 p.

Herce Vallejo, M. 1983. «La utilización de indicadores topológicos en el analisis de redes de communicaciones - Ensayo sobre la red de carreteras de Cataluña ». Documents d'Anàlisi Geogràfica, $n^{\circ} 3: 3-46$

Herrmann, P. 2007. Itinéraires des voies romaines. Paris, Editions Errance, 275 p.

Hodson, F.R. 1971. "Cluster analysis Numerical typology and prehistoric archaeology », in : F.R. Hodson, D.G. Kendall et Tautu P. (éds), Mathematics in the Archaeological and Historical Sciences. Proceedings of the Anglo-Romanian Conference, Mamaia 1970, organized by The Royal Society of London, and The Academy of the Socialist Republic of Romania, Edinburgh, Edinburgh University Press : $30-45$.

HuRe, A. 1978. Le Sénonais gallo-romain, Bruxelles, Editions Culture et civilisation, 422 p.

Huriot, J.-M., Perreur, J. 1994. « La centralité / L'accessibilité », in : (Auray J.-P. et al. 1994) : 4758.

InGRAM, D.R. 1971. "The concept of accessibility: a search for an operational form ». Regional Studies, $5: 101-107$

JEANNET, N. (à paraître). « Contributions pratiques d'une géostatistique raisonnée en environnement : méthodes et application à la cartographie régionale de la pollution par le dioxyde d'azote $\left(\mathrm{NO}^{2}\right)$ en Alsace », in: Aubry, L. et Soulier, P. (dir.): Les approches spatiales des méthodes statistiques appliquées à l'archéologie. Journée d'étude organisée par le 13 décembre 2007 à la MAE de Nanterre. Cahiers transversaux de l'UMR 7041

Johnson, A.W. et Earle, T. 1997, 2000 (2e éd). The Evolution of Human Societies. From Foraging Group to Agrarian State. Stanford, Stanford University Press, 456 p.

JoLy O. 1999. Geographical position : State of french art of spatial accessibility indicators. SPESD France, Groupe de travail I. 1, 23 p. 
Journées intenationales d'histoire. Centre culturel de l'abbaye de Flaran, 1982. «L'homme et la route en Europe occidentale au Moyen Age et aux Temps Modernes », in : Cahier de Flaran, $n^{\circ} 2$, Auch. 303 p.

Jullian, C. 1920. Histoire de la Gaule, tome V, Paris Hachette, 381 p.

Jung, C. 1999. Morphogénèse, fonctions et évolution de la centuriation B d'Orange et essai de restitution diachronique des paléopaysages du Tricastin (Drôme-Vaucluse). Thèse de doctorat, Université de Tours, $366 \mathrm{p}$.

Konvirz J.F. 1987. Cartography in France, 1660-1848, Science, Engineering and Statecraft, Chicago/ London, University of Chicago press. 194 p.

Laffont, P. Y et Bréchon, F. 2008. «Châteaux, incastellamento et réseau viaire au Moyen-Age en France méridionale », in : Conpatangelo-Soussignan R., Bertrand J. R., Chapman J. et Laffont P. Y. (dir.). Marqueurs des paysages et systèmes socio-économiques. Actes du colloque COST du Mans (7-9 décembre 2006), Rennes, Presses Universitaires, 2008 : 149-163.

Lамвот, B. 1996. «Les Rèmes à la veille de la romanisation. Le porcien au Ier siècle avant J. C. », in : Bayard D. et Collard J. L. (dir.), Revue Archéologique de Picardie, $n^{\circ}$ spécial, 11/1996, Amiens : 1338.

Lavigne, C. 1997. «Parcellaires de fondation et parcellaires de formation à l'époque médiévale en Gascogne. Clefs de lecture et problèmes d'interprétation », in : Chouquer G. (dir.) 1997 Les Formes du paysage, Tome 3 : L'analyse des systèmes spatiaux, Paris, Editions Errance : 149-159.

LebecQ, S. 1989. « La Neustrie et la mer », in : H. Atsma (publ.), La Neustrie, Les pays au nord de la Loire de 650 à 850. Jan Thorbecke Verlag Sigmaringen, 1989 : 406-440.

Legros, R. 1983. «Les chemins des Rèmes », in : Chevallier R. (dir.) 1983 Les voies anciennes en Gaule et dans le monde romain occidental, Colloque tenu à Paris, ENS, 5-6 juin $1982 »$. Caesarodunum $n^{\circ}$ XVIII, Tours, Université de Tours : 269-289.

Leone, F., Aste, J.-P., Velasquez, E. 1995. «Contribution des constats d'endommagement au développement d'une méthodologie d'évaluation de la vulnérabilité appliquée aux phénomènes de mouvements de terrain ». Bulletin de l'Association de Géographes Français - « Croissance urbaine et risques naturels 》, Vol. 4, 72ème année : 350-371.

LePETIT, B. 1984. Chemins de terre \& voies d'eau, réseaux de transport organisation de l'espace, Éds. 
de l'E.H.E.S.S., Paris. 148 p.

Lepetit, B. 1988. Les villes dans la France moderne (1740-1840), Paris, Albin Michel. 490 p.

LeturcQ, S. 1997. «La route et le paysage. Dynamique et stabilité des réseaux routiers beaucerons entre Etampes et la forêt d'Orléans », in : Chouquer G. (dir.) 1997 Les Formes du paysage, Tome 3 : L'analyse des systèmes spatiaux, Paris, Editions Errance: 66-78.

Leurent, F. 2006. Structures de réseau et modèles de cheminement. Lavoisier, 394 p.

Levy, J. et Lussault, M. (dir.), 2003. Dictionnaire de la géographie et de l'espace des sociétés. Paris, Belin, $1033 \mathrm{p}$.

Livet, G. 2003. Histoire des routes et des transports en Europe. Des chemins de Saint-Jacques à l'âge d'or des diligences. Strasbourg, Presses universitaires, 608 p.

LLobera, M. 2003. "Estending GIS-based visual analysis: the concept of visualscapes 》. International Journal of Geographical Information Science Vol. 17/1 : 25- 48.

Lot, F. et FAwTIER, R. 1932. Le premier budget de la monarchie française, Paris, 259 p.

Loubès, G., « Routes de la Gascogne médiévale », Journées intenationales D’histoire. Centre culturel de L'abbaye de Flaran, 1982. "L'homme et la route en Europe occidentale au Moyen Age et aux Temps Modernes », in : Cahier de Flaran, $n^{\circ} 2$, Auch : 33-56.

Luchaire, A. 1885. Les actes de Louis VII, Paris, Editions Alphonse Picard, 527 p.

Lundmark, H. 1984. « The identification of tribal hierarchies », in : K. Kritiansen (éd.), Settlement and Economy in Later Scandinavian Prehistory, British Archaeological Reports International, Series 211 : 43-62.

Malrain, F., Matterne, V., Méniel, P. 2002. Les paysans gaulois. Paris, Errance, 2002, 236 p.

Marchand, C. 1997. «Réseau viaire et dessin parcellaire : étude morphologique de la région du Gâtinais oriental. » In Chouquer (dir.) 1997, Les Formes du paysage, Tome 3 : L'analyse des systèmes spatiaux, Paris, Editions Errance, 66-77.

Marchand, C. 2000. «Recherches sur les réseaux de formes. Processus dynamiques des paysages du sénonais occidental », thèse de doctorat, Université de Tours. 2 vol. 
Marchand, C. 2003. «Des centuriations plus belles que jamais ? Proposition d'un modèle dynamique d'organisation des formes », Études Rurales, juillet décembre 2003, n 167-168 : 93-114.

Matнis, Ph. (dir.) 2003. Graphes et réseaux, modélisation multiniveau. Paris, Lavoisier, 361 p.

MAzoyer, M. et Roudart, L. 1997, 2002 (rééd). Histoire des agricultures du monde : du néolithique à la crise contemporaine, Paris, Seuil, 705 p.

Mennessier-Jounannet, C. et Buchsenschutz, O. 1996. " Des " champs celtiques » aux systèmes de parcellaire protohistoriques », in : Chouquer G. (dir.) 1996. Les Formes du paysage, Tome I : études sur les parcellaires : pré-actes du colloque d'Orléans (mars 1996). Paris, éditions Errance : 174-180.

Ministère de L'Environnement et du CAdre de Vie 1980. Lutte contre les inondations - Méthode sommaire d'évaluation des critères économiques. Direction de la Prévention des Pollutions - Service de l'Eau - Sous-Direction des Eaux Continentales, 75 p. annexes

Mordant, C., Saligny, L. Poulet-Crovisier, N. «La circulation des objets métalliques à l'âge du Bronze entre Rhône et Saône: utilisation d'une transformation cartographique linéaire », Mappemonde $n^{\circ} 83$ (3-2006), en ligne sur http://mappemonde.mgm.fr.

Muraco, W.A. 1972. « Intraurban accessibility ». Economic Geography, 48 : 388-405.

Nordman, D. 1990. « La connaissance géographique de l'État (XIV $-\mathrm{XVII}{ }^{\mathrm{e}}$ siècles) », in L'État moderne : le droit, l'espace et les forme de l'État, Paris, CNRS, 199 : 175-188.

Nuninger, L. et SANDERs, L. 2006. «La modélisation des réseaux d'habitat en archéologie: trois expériences », Mappemonde ${ }^{\circ} 83$ (3-2006), en ligne sur http://mappemonde.mgm.fr.

Ouzoulias, P. 1991. «Eudes Rigaud et le vieux chemin Paris-Rouen », in : Cuisenier J. (dir.), 1991. Matière et figure. Paris, La Documentation française, Collection études et Travaux n³ : 17-42.

Ozouf-Marignier M.-V. et Verdier N. 2000. "L'événement: un objet historique à emprunter", L'Espace Géographique, n³, 218-223.

Parish, Y.I.H., Müller, P. 2001. «Procedural Modeling of Cities», in : Proceedings of ACM SIGGRAPH 2001, ACM Press / ACM SIGGRAPH, New York. E. Fiume (ed), Computer Graphics, Annual Conference Series, ACM : 301-308.

Pelletier, M. 2002. Les cartes des Cassini, La science au service de l'État et des régions, Paris, 
CTHS. 338 p.

Pérol, C. 2004. « Cheminement médiéval, l’homme l'historien et la route », in Fray, J.L. et Pérol, C. L'historien en quête d'espaces, Clermont-Ferrrand, Presses Universitaires Blaise Pascal : 91-107.

Petitfrère C. 2003. «Dessine-moi un village... Le « village» sur la carte et le plan en Limousin sous l'Ancien Régime », in Tricard Jean (dir.), Le Village des Limousins : Études sur l'habitat et la société rurale du Moyen Âge à nos jours, Limoges, Presses universitaires de Limoges. 257-272.

Picon, A. 1992. L'invention de l'ingénieur moderne : l'École des ponts et chaussées, 1747-1851, Paris, Presses de l'École des ponts et chaussées. 767 p.

Picon, A. et Yvon, M. 1989. L'ingénieur artiste, Dessins anciens de l'École des ponts et chaussées, Paris, Presses de l’École des ponts et chaussées. 206 p.

Pinchemel P. 1944. « Habitat Rural et Maisons Rurales en Picardie», Bulletin de l'association des géographes français, ${ }^{\circ}$ 163-166. 102-108.

Pinchemel, P. et Pinchemel, G., 1988. La face de la terre, éléments de géographie. Paris, Armand Colin, $515 \mathrm{p}$.

Pion, P. 1990. « De la chefferie à l'État ? Territoires et organisation sociale dans la vallée de l'Aisne aux Âges des métaux (2200-20 av. J-C.) », Archéologie et Espaces, Actes des Rencontres Internationales d'Archéologie et d'Histoire d'Antibes (10 ; 1989), Editions APDCA, Antibes : 183260.

PIтTs, F. 1965. « A graph theoretic approach to historical geography ». The Professional Geographer, $\mathrm{n}^{\circ} 17: 15-20$.

Prou, M. 1884. Les coutumes de Lorris, Paris, 176 p.

Revel, J. 1992. « La région », in, Les lieux de mémoire, III, Les France, 1, Conflits et partages, nrf, Gallimard : 850-883.

Robert, S. (à paraître). «Des outils pour l'analyse de la transmission des trames et réseaux planimétriques : approches multiscalaires et traitements sur SIG», in : direct Colloque du réseau thématique pluridisciplinaire «Paysage et environnement, de la reconstitution du passé aux modèles prospectifs », Chilhac 27-30 sept. 2006.

Roвert, S. 1996. «Le Parcellaire du plateau de Sénart (Seine-et-Marne)», in : Chouquer G. (dir.) 
1996 : Les Formes du paysage, Tome I : études sur les parcellaires : pré-actes du colloque d'Orléans (mars 1996). Paris, éditions Errance : 11-27.

Robert, S. 1997. "Le relevé parcellaire sur photographies aériennes et cartes anciennes ", in : Chouquer G. (dir.) 1997. Les Formes du paysage, Tome 3 : L'analyse des systèmes spatiaux, Paris, Editions Errance : 88-95

Robert, S. 2004. L'analyse morphologique des paysages entre archéologie, urbanisme et aménagement du territoire. Exemples d'études de formes urbaines et rurales dans le val d'Oise, thèse de doctorat, Université de Paris 1, 2 vol.

Robert, S. 2006a. « La résilience des réseaux routiers : l'exemple du Val-d'Oise », in : Bulletin AGER $n^{\circ} 15$, année 2005, Besançon, Presses Universitaires de Franche-Comté, 2006 : 8-14.

RoBert, S. 2006b. «Les itinéraires anciens traversant le département du Val-d'Oise », in : Bulletin archéologique du Vexin Français et du Val-d'Oise, $n^{\circ} 38: 7-23$

Robert, S. 2007. "Modeling Ancient Roads », in : Medieval Europe Paris 2007, 4e Congrès international d'Archéologie Médiévale, Institut National d'Histoire de l'Art, Paris, 3-8 septembre 2007. en ligne sur http://medieval-europe-paris-2007.univ-paris1.fr.

Roncayolo, M. 1988. "la morphologie, entre la matière et le social", Villes en parallèle, $\mathrm{n}^{\circ} 12-13,42-$ 59.

Rouche, M. 1982. "L'héritage de la voirie antique dans la Gaule du haut Moyen-Age (Ve-XIe siècle) », in : Flaran, 1982. "L'homme et la route en Europe occidentale au Moyen Age et aux Temps Modernes », in : Cahier de Flaran, $n^{\circ} 2$, Auch. : 12-32.

SAHLINs, P. 1996. Frontières et identités nationales, la France et l'Espagne dans les Pyrénées depuis le XVIIe siècle, Paris, Belin ( $1^{\mathrm{er}}$ ed. en anglais 1989), $416 \mathrm{p}$.

Sakarovitch, M. 1984. Optimisation combinatoire. Méthodes mathématiques et algorithmiques. Programmation discrète, Paris, Hermann, 269 p.

SAn JuAn, G. 2005. « La Normandie dans l'âge du Bronze européen », in: C. Marcigny, C. Colonna, E. Ghesquière et G. Verron (dir.), La Normandie à l'aube de l'histoire, Les découvertes archéologiques de l'âge du Bronze 2300-800 av. J.-C.. Paris, Somogy : 17.

Schnapp A. 1993. La conquête du passé. Aux origines de l'archéologie. Paris, éditions Carré, 511 p. 
SHefFI, Y. 1985. Urban Transportation Networks - Equilibrium Analysis with Mathematical Programming Methods. Prentice Hall, 400 p.

Spratt, D.A. 1981. "Prehistoric boundaries on the North Yorkshire Moors », in: G. Barker (éd), Prehistoric communities in Northern England : Essays in social and Economic reconstruction, Sheffield, University of Sheffield Press : 87-104.

TissieR, J.-L. 2003. « Chemins et géographie », Les carnets du paysage, $\mathrm{n}^{\circ} 11: 35-53$

Touzery M. 1995. Atlas de la généralité de Paris au XVIII siècle : un paysage retrouvé, Paris, Comité pour l'histoire économique et financière de la France. 176 p.

Van Leusen, P. M. 2002. Pattern to process : methodological investigations into the formation and interpretation of spatial patterns in archaeological landscapes. Groningen, Rijkuniversiteit. 365 p.

Verdier N. 2002. «Poste et territoires : évolution de la pensée du territoire chez les administrateurs de la Poste au XIXème siècle », in : Le Roux (dir), Histoire de la poste. De l'administration à l'entreprise, Editions rue d'Ulm : 61-86.

VERDIER N. 2004. "L'échelle dans quelques sciences sociales : petite histoire d'une absence d'interdisciplinarité », in O. Orain, D. Pumain, C. Rozemblat et N. Verdier, Géographie, échelles et temporalités en géographie, Paris, CNED : 25-56.

Verdier N. 2007. « Le réseau technique est-il un impensé du XVIIIe siècle : le cas de la poste aux chevaux », Flux, Cahiers scientifiques internationaux Réseaux et Territoires, 68 : 7-21.

VERDIER N. 2008a. « Gerarchie urbane e città in rete : l'urbanizzazione della Francia tra 1700 e 1830 », in Iachello E. e Militello P. (dir.), L'insediamento nella Sicillia d'étà moderna et contemporanea, Bari, Epuglia.

Verdier N. 2008b. "Modeler le territoire : les ingénieurs des Ponts et chaussées et leurs usages de la carte (fin XVIIe-début XIXe siècles)", in Isabelle Laboulais (dir.), Les usages des cartes (XVIIe-XIXe siècles, pour une approche pragmatique des productions cartographiques, Strasbourg, Presses universitaires de Strasbourg, 2008, 51-66 et VI-VII.

Verhagen P., Gili S., Mico R., Risch R. 1999. « Modelling Prehistoric Land Use Distribution in the Rio Aguas Valley (SE Spain) », in: Dingwall L. et al. (eds) : Archaeology in the Age of the Internet. Proceedings of the CAA97 conference, Oxford, BAR International Series, 750, CDRom. 
Vidal De Lablache, P., 1902. "Routes et chemins de l'ancienne France », Bulletin de géographie historique et descriptive, $\mathrm{n}^{\circ} 17: 115-126$.

Vidal, L., Pomarèdes, H. et Sauvage, L. 1996. «Traces matérielles de la mise en valeur et de l'aménagement des campagnes aux portes de Nîmes : les parcellaires antiques de la plaine du Vistre », in : Chouquer G. (dir.): Les Formes du paysage, Tome II : études sur les parcellaires : actes du colloque d'Orléans (mars 1996). Paris, éditions Errance, 1996 : 57-66.

VIGNON E. 1862. Études sur l'administration des voies publiques en France aux dix-septième et dixhuitième siècles, Paris, Dunod. 3 vol. XIII-146-145, 213-358, 135-281 p.

Vion, E. 1989. «L'analyse archéologique des réseaux routier : une rupture méthodologique, des réponses nouvelles », Paysages découverts $I:$ 67-69.

Vita-Finzi, C. et Higgs, E.S. 1970. « Prehistoric economy in the Mount Carmel area of Palestine: site catchment analysis », Proceedings of the Prehistoric Society, 36 : 1-37.

Voyages et Voyageurs au Moyen Age, 1996. Société des historiens médiévistes de l'enseignement supérieur public. Voyages et Voyageurs au Moyen Age. XXVIe Congrès de la SHMES (LimogesAubazine, mai 1995), Paris, Publications de la Sorbonne, 314 p.

Wabont, M., Abert, F., Vermeersch, D. 2006. Le Val-d'Oise - 95. In : Provost M. (dir.), Carte Archéologique de la Gaule. Le département du Val-d'Oise. Paris : Editions Les Belles Lettres, 495 p.

Wasserman, S., Faust, K. 1994. Social Network Analysis - Methods and applications. Cambridge University Press, $857 \mathrm{p}$.

Watteaux, M. 2004. «Le plan radio-quadrillé des terroirs non planifiés », in : Chouquer G. (prés.). Etudes Rurales $n^{\circ} 167-168.2004$ : 187-214.

Weber, J. 2000. «Using GIS to Model and Visualize Congestion Effects on Individual Accessibility », in : Proceedings of the Annual Meeting of the East Lakes and West Lakes Divisions of the Association of American Geographers, Oxford, Ohio, 18 p.

Zaksek, K., Fovet, E., Nuninger, L. et Podobnikar T. 2007. " Path modelling and settlement pattern », in : Computer Applications and Quantitative methods in Archaeology, Berlin, , Germany, April 2-6, 2007. Bonn (Germany): Habelt (Kolloquien zur Vor- und Frühgeschichte, vol. 10. 